\title{
Survivin Clinical Features in Cervical Cancer
}

\author{
Miftakh Nur Rahman ${ }^{1,2}$, Maria Novalentina ${ }^{1,2}$, Chyntia Resti Wijaya ${ }^{1,2}$ \\ ${ }^{1}$ Faculty of Pharmacy, Padjajaran University, Bandung, Indonesia \\ ${ }^{2}$ Prodia Clinical Laboratories, Jakarta Indonesia
}

Cervical cancer is the primary lethal malignancy for women worldwide, but because it develops over time, it would be one of the most preventable types of cancer. Dysregulation of apoptosis in cells plays a critical role in the malignancy development. Survivin is the smallest inhibitor apoptotic protein (IAP) which has an important part in regulating cell division and inhibitor of apoptosis. This review focused on survivin features in cervical cancer from mechanisms of malignancy relationship to human papillomavirus (HPV) infection through E6 oncogenic protein, role as a biomarker in diagnosis, prognosis, staging and prediction of metastasis, and also as a target for therapy. Regulation of survivin divided into two main groups; cell cycle dependent and cell cycle independent pathway to maintain life and death balance. Survivin expression is upregulated by E6 protein simultaneously repressing p53. Thus cancerous cervical tissue developed. Survivin is also upregulated in hypoxia, a common condition in many tumors and increased angiogenesis. Survivin plays a significant role in chemotherapy and radiation resistance in many cases of cervical cancer. As a target of therapy, survivin has a promising performance, suggested very specific, no issue of resistance and also enhance the effectiveness of chemo and radiation therapy. The goal of treatment is to lower survivin expression through transcription inhibition, immunotherapy based on cytotoxic $\mathrm{T}$ lymphocytes (CTL) activity and gene therapy.

Keywords: cervical, cancer, survivin, HPV E6, therapy

\section{Introduction}

\section{Cervical cancer}

Cervical cancer is the primary lethal malignancy for women worldwide, but because it develops over time, it would be one of the most preventable types of cancer. ${ }^{1,2}$ Cervical cancer is the most easily cured cancer if detected as soon as possible. Cervical cancer started from the lower part of the uterine and happened when the cells proliferate out of control. The primary cause of cervical cancer is high-risk type human papilloma virus (HPV). ${ }^{2}$
HPV infection and transmission in human passed through genital contacts. ${ }^{2,3}$ If the HPV infection became persistent, it will develop cancer over time. ${ }^{2}$ Several types of HPV have identified in aggressive cervical cancer, whereas others are associated with dysplasia or carcinoma in situ. The known 13 types of high-risk HPV (HR-HPV) are 16, $18,31,33,35,39,45,51,52,56,58,59$ and $68 .^{2,3}$ Until now, the assessment of prognosis cervical cancer seen from a tumor marker. Clinico-histopathological tumor developed to determine prognostic factors for cervical cancer as viewed antigen serum, angiogenesis factors and apoptosis factor. ${ }^{4}$

Date of submission: December 12, 2016

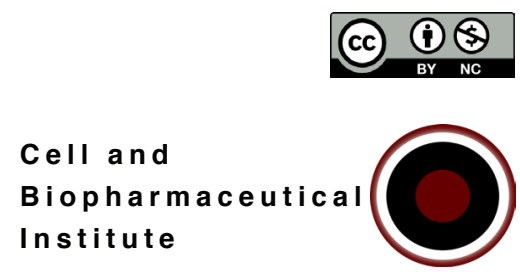


Dysregulation of apoptosis in cells plays a significant role in the development of cancer malignancy. ${ }^{5}$

\section{Pathophysiology of cervical cancer}

The development of oncogenic HPV infection into cervical cancer occurs if the infection settled. The development of abnormal cells in cervical epithelium can develop into precancerous also called cervical intraepithelial neoplasia (CIN). ${ }^{1}$ Developmentally abnormal cells become cancerous is following:

1. CIN I/low-grade squamous intraepithelial lesions (LSILs): in this stage, there is a change that cells infected with oncogenic HPV will make new virus particles.

2. CIN II/high-grade squamous intraepithelial lesions (HSILs): the cells continued to show symptoms of abnormal precancerous.

3. CIN III: surface layer of the cervix filled with the abnormal cells and may involve the full thickness. CIN III is usually caused by certain types of HPV. If not treated, these abnormal cells become cancer and infected normal tissues.

4. Invasive cervical cancer.

\section{Types of cervical cancer}

Classification of cervical cancer and pre-cancer determined by how they appearance on the microscope. The major types of cervical cancer are squamous cell carcinoma (SCC) and adenocarcinoma. SCC developed from exocervix cells and began the abnormal transformation within the joints of endocervix and exocervix area. On the other hand, adenocarcinoma developed from gland cells which produce mucus in the endocervical area. SCC considered as the most commonly cervical cancer, but in the past 30 years, adenocarcinomas become more frequent. Another type but less common, mixed of squamous cell carcinoma and adenocarcinoma, termed as adenosquamous carcinoma. ${ }^{1}$

\section{Survivin}

To inhibit the growth of cancer cells from invading normal cells should be quick and appropriate. The standard treatments such as surgery, chemotherapy and radiotherapy have yielded significant results in inhibiting the growth of cancer cells. But the development of drug resistance and the occurrence of cancer metastasis remains a challenge that is difficult to control. ${ }^{6}$ Immunotherapy is one of the type cancer therapy designed to trigger adaptive immune system to fight cancer and prevent metastasis. Normal cells will die by itself if it suffered damage to its deoxyribonucleic acid (DNA), but not so in cancer cells; they do not have the ability to detect defects and resistance to apoptosis. The ability of cancer to escape from the immune system is the one of primary evasion mechanism, and at the same time, they can promote their proliferation. ${ }^{6}$ Survivin found overexpressed in cancer cells which suggested to have a significant role in the regulation of T-cell responses and becomes a potential for targeted cancer therapy. ${ }^{7}$ On the other hand, survivin has a role in angiogenesis. Thus, survivin promotes cell proliferation and migration in the survival of cancer from apoptosis. $^{8}$

Survivin also called baculoviral inhibitor of apoptosis repeat-containing 5 (BIRC5), which is the smallest inhibitor of apoptosis protein (IAP) N-terminus with a single baculovirus IAP repeat (BIR) domain and the C-terminus coiled coil (CC) domain. ${ }^{7}$ Survivin has 142 amino acids and molecular weight of $16.5 \mathrm{kDa}$. Compared with other IAPs, Survivin has an important role in regulating cell division and inhibitor of apoptosis. In the cell cycle, survivin expressed at the Gap 2/mitosis (G2/M) phases. ${ }^{5}$ This condition makes survivin is potential because it can be a promising therapy for cancer. The expression of survivin in CIN I, CIN II and CIN III had increased significantly, suggested that survivin could be a useful marker for the diagnosis and differentiate the value of CIN patients. ${ }^{9}$ Survivin expression also correlated with the patient's clinical stage. ${ }^{6,9}$ Survivin expression in tumors is also associated with a high rate of disease recurrence and resistance to therapy, and it confers a significant survival advantage to tumor cells. ${ }^{10}$

\section{Expression regulation of survivin}

Dysregulation of survivin is a key of many types of cancer. Survivin expression associated with an unusual activation of some receptor tyrosine kinases (RTKs), for example, epidermal growth factor receptor (EGFR), erythroblastosis oncogene B (ErbB-2)/human epidermal growth factor receptor 2 (HER2), insulin-like growth factor-1 (IGF-1) and IGF-1 receptor (IGF-1R). Other receptors including viability signal cascades of cells (phosphatidylinositol-3kinase and protein kinase $\mathrm{B}$ or PI3K/Akt), mammalian target of rapamycin (mTOR), signal transducer and activator of transcription (STAT), hypoxia-inducible factor-1 (HIF-1), etc. ${ }^{11}$ Different regulation of survivin expression occurs in cycle-dependent pathways; there are transcriptional level and post-translational regulations and cell cycleindependent pathway. 


\section{Cycle-dependent pathway}

\section{a. Transcriptional level regulation}

Survivin genome sequences contain a cell cycle-dependent element $(\mathrm{CDE}) /$ cell cycle genes $(\mathrm{CHR})$ in the promoter region, showing that survivin can be cell cycle-regulated gene. Many kinds of micro-ribonucleic acid (miRNA) are identified to regulate survivin's expression via binding to 3'-untranslated (UTR) region of survivin's messenger RNA (mRNA), so that produce protein translational changes leading to the mRNA degradation.

miRNA is non-encoding small RNA expressing various post-transcriptional target genes. Downregulation of miRNA-218 (miR-218) has reported in some dangerous human cells including cervical cancer, lower miRNA-218 serum level that has been described due to tumor invasion. ${ }^{12}$

\section{b. Post-translational level regulation}

Survivin level influenced by protein modifications like phosphorylation and polyubiquitination. Survivin phosphorylation at threonine 34 (Thr34) by cell division cycle protein 2 (CDC2), which also called cyclin-dependent kinase 1 (CDK1) has an effect of stabilizing surviving during prometaphase and metaphase of proteasomal degradation and prolong anti-apoptotic threshold in the cells which undergo mitosis. Survivin's phosphorylation at Thr48 has an effect to change the binding with borealin and thereby affects its ability to enhance cell proliferation and inhibits cell death. ${ }^{13}$

The ubiquitin-proteasomal pathway is also identified to regulate survivin's degradation in a cell cycle-dependent manner and BIR domain of surviving essential to keep its stabilization at $\mathrm{G} 2 / \mathrm{M}$ phase on post-translation.

\section{Cycle-independent pathway}

Cycle-independent mechanism affecting survivin's expression level involves hematopoietic response and cytokine vascular remodeling, STAT3-dependent signal transduction and PI3K activity, angiopoietin-1 (ANG1), interleukin 11 (IL-11), STAT3 signal transducer and transcription activator. ${ }^{11}$

Survivin expression associated with the unusual activity of several RTKs, such as EGFR, HER2, HER3 and IGF-1. These kinases can trigger various viability signal cascades like P13K/Akt-mTOR organizing survivin's expression. Phosphorylated serine 81 (Ser81) survivin induce activity of protein kinase A (PKA) in the cryoprotection mechanism via P13K. ${ }^{14}$

\section{Molecular mechanism of survivin in tumorigenesis}

Survivin involved in tumorigenesis through some different mechanisms including apoptotic inhibition, cytokinesis regulation, cell cycle progression and the participation in many kinds of pathway like p53 signaling, wingless-related integration site (Wnt), transforming growth factor (TGF) and Notch pathways. ${ }^{\text {? }}$

\section{Survivin's mechanism in apoptotic pathway}

As an IAP, survivin protects cells against apoptosis by inhibits caspase. However, survivin isn't able to bind and directly inhibits caspase rather interacts with pro- and antiapoptotic proteins. Thereby survivin should form a complex with X-linked IAP (XIAP), an IAP directly binding caspase, resulting in increased XIAP stability and to proteasomal degradation.

There are two apoptotic mechanisms known in mammals: intrinsic and extrinsic apoptotic. These both pathways are executed by some caspase proteins, such as initiator caspase (caspace-8 and -9) and executioner caspase (caspase-3, -6 and -7). Apoptosis initiated by cytochrome c binding cardiolipin within the inner mitochondrial membrane and activating caspase-9. Caspase-9 further activates caspase- 3 and -7 which are responsible for cells damage. Survivin overexpression associated with apoptotic inhibition initiated by extrinsic and intrinsic apoptotic pathways. An in vitro study indicates that survivin phosphorylation at $\mathrm{Thr} 34$ and $\mathrm{CDC} 2$ is necessary for survivin interaction with caspase-3, -7 and -9 . Several further studies found that a survivin mutation at Thr34 can induce cytochrome c release from mitochondria leading to apoptosis. Phosphorylated survivin at Thr34 by CDK1 can protect cells against cell death by inhibiting caspase- 9 during mitosis. ${ }^{15}$ Beside Thr34, Ser81 is also reported to enhance cell protection, although treated by tumor necrosis factor (TNF)-related apoptosis-inducing ligand (TRAIL). ${ }^{16}$

The gene that encodes a protein survivin is BIRC5 have the 17q25 locus will affect apoptotic pathways by action on the caspases and increase cell division. Survivin in cervical cancer can inhibit apoptosis through two pathways. The first path is to upgrade with CDK4 as well as the release of $\mathrm{p} 21$. The second pathway is by inhibiting the activity of caspase-7 and caspase- 3 . $^{13,17}$

Functional study on cervical cancer showed that by suppressing survivin expression in HeLa cells causing 
spindle impairment and promoting apoptosis. ${ }^{8}$ Disruption of the expression and function of apoptotic inhibitor survivin cause caspase-dependent cell death during the G2/M phase of cell cycle and cell division impairment indicated by centrosome dysregulation, multipolar and nuclear mitotic spindle, polyploidy cells. ${ }^{19}$ Anti-apoptotic function conducted by cytoplasm survivin, whereas nuclear survivin is involved in cell division setting. ${ }^{20}$

\section{Survivin's mechanism in cell cycle}

In several studies noted that survivin expression regulated by cell cycle and loops during the G2/M phase, and down fast during the G1 phase. Survivin is dynamically located in the different region of the chromosome during the cell cycle. As long as mitosis, survivin serves as a part of chromosomal passenger complex (CPC). ${ }^{21}$

$\mathrm{CPC}$ is consists of a localization module and a kinase module, which connected by inner centromere protein (INCENP) center. Localization module composes INCENP $\mathrm{N}$-terminal and borealin as well as surviving C-terminal, leads first CPC into centromere, then mitotic spindle and ultimately into midbody. Survivin is responsible for leading $\mathrm{CPC}$ into centromere at early prophase. The BIR domain binds the N-terminal of phosphorylated histone $\mathrm{H} 3$ at $\mathrm{Thr} 3$; a mitotic-specific phosphorylation clearly marks the inner centromere. ${ }^{22}$

Survivin accumulates in the centromere during the G2 phase and then begins to diffuse with the chromosome arms and abundant in the middle of centromere during prophase and metaphase in mitosis. In anaphase, survivin is localized at $\mathrm{mid} / \mathrm{center}$ of spindle because it failed to bind the centromeres. During cytokinesis, survivin concentrated in the middle. Cell cycle-specific regulation of surviving noted that survivin plays a significant role in cancer cell proliferation. Also, survivin could be phosphorylated by CDK1 which is a critical kinase for cell cycle regulation. ${ }^{23}$

\section{Survivin mechanism in cancer}

Survivin overexpression in cancer is related to mutation inside the promoter, gene duplication or dysregulation of related transcriptional factors. P532, for example, functionally inactivated by mutations in several types of cancer, seems to suppress survivin's mRNA transcription. Nuclear factor-kappa B (NF- $\mathrm{KB})$ induction and avian myelocytomatosis virus oncogene cellular homolog (c-Myc) expression via P13K/Akt pathway are also leading to survivin overexpression in a cell cycle-independent manner in tumor cells. ${ }^{24}$
Survivin aberrant expression and p53 wildtype disruption associated with tumorigenesis. There is a significant possibility that survivin functionally binds p53. Many studies indicated that p53 wildtype could also suppress survivin expression in transcriptional level and the loss of survivin function partially mediates p53-dependent apoptotic pathway. Further analysis via chromatin deacetylation of survivin promoter shows that chromatin modification can contribute in surviving expression silencing by $\mathrm{p} 53 .{ }^{8}$

Survivin facilitates cancer cells defense and proliferation, thus increase angiogenesis, a process providing oxygen as well as a nutrient for emerging unavoidable tumor which allows the tumor growth beyond the certain size. Survivin enhances angiogenesis by stimulating tumor cells to express and secrete increased number of vascular endothelial growth factor (VEGF) which is an essential signaling molecule in all angiogenesis step. P13K/Akt signaling and $\beta$-catenin-T-cell factor/lymphoid enhancer factor (TCF/LEF)-dependent transcriptions were involved in many related cancer genes expression, including survivin and VEGF. ${ }^{25}$

Survivin expression is not only upregulated in cancer cells but also in tumor vascular endothelial cells. In the tumor, IAP survivin had pleiotropic function and associated with tumor progression, metastasis and angiogenesis. The mechanism includes interaction and stabilization of complex anti-apoptotic protein XIAP and pro-apoptotic protein inhibition such as second mitochondria-derived activator caspases (SMAC)/direct inhibitor of apoptosis-binding protein with low pI (DIABLO) or apoptotic inducing factor (AIF). Recently, this survivin promotes invasion and metastasis by increasing NF- $\mathrm{KB}$ cells activated by NF$\kappa \mathrm{B}$-dependent transcription of fibronectin. Intramolecular between XIAP and survivin triggers tumor cell invasion and increases metastasis. This pathway is independent of IAP inhibition of cell death. On the contrary, a complex survivin-XIAP activates NF- $\kappa \mathrm{B}$, which in turn causes increased fibronectin gene expression, signaling by integrin beta- 1 and motility cell activation by focal adhesion kinase (FAK) and sarcoma (Src) kinase. ${ }^{25}$

\section{Survivin mechanism in Wnt/B-catenin}

During tumorigenesis, $\beta$-catenin accumulation in the cytoplasm known as Wnt signaling pathway hallmark. Then Wnt signaling induces $\beta$-catenin translocation into the nucleus to form $\beta$-catenin factor/TCF transcription 
activator conducts upregulation of target genes such as survivin, c-Myc and VEGF. Increased survivin expression then protects the cells from apoptosis and increase cancer cell proliferation. ${ }^{26}$

Survivin expression associated with increased $\beta$-catenin/TCF-LEF reporter activity via P13K/Aktdependent mechanism. Thereby, increased expression and many target genes including VEGF accumulation occurs, which will promote angiogenesis. The connection between those markers involved in angiogenesis illustrated in Figure 1. Casein kinase 2 (CK2) also promotes tumor cell's viability and inhibits apoptosis by involving increased $\beta$-catenin/ TCF-LEF transcriptional genes. ${ }^{27}$

\section{Survivin mechanism in hypoxia signaling}

Hypoxia contributes to various biological processes such as angiogenesis, malignancy, metastasis and therapy resistance. While in response to hypoxia, the cells undergo gene expression change initiated by the HIF-1 transcriptional factor, which is heterodimeric protein complex consists of HIF- $1 \alpha$ and HIF- $1 \beta$ responsible of metabolic adaptation, $\mathrm{pH}$ controlling and neovascularization process. ${ }^{28}$

HIF- $1 \alpha$ protein expression is negatively regulated in cells nonhypoxic by ubiquitination and proteasomal degradation. In hypoxic conditions, the levels of HIF-1 $\alpha$ protein will increase dramatically. Overexpression of HIF$1 \alpha$ may occur in conditions of intratumoral hypoxia and genetic changes that activate the transcription of genes involved in cancer biology, glucose metabolism, cell proliferation, apoptosis, invasion and prognosis of cancer.

Hypoxic conditions lead to increased activation of survivin promoter, wherein the first $158 \mathrm{bp}$ of survivin gene promoter sequence into port responsive to hypoxia. Several mechanisms between HIF-1 and survivin in hypoxia, among others:

1. HIF-1 can bind to other DNA sequences directly in the promoter region of survivin,

2. HIF-1 binding indirectly through interactions with transcription factor protein proteinuria as activator protein 1 (AP-1), specificity protein $1(\mathrm{Sp} 1)$ and the effects of c-Myc indirectly affect the expression of the target gene HIF-1,

3. HIF-1 induces the expression of other factors that stimulates transcription of survivin transcription. Sp1/ $\mathrm{Sp} 3$ and NF-kB promoter binding surviving that interact with HIF-1 to regulate the expression of survivin..$^{29}$
Reactive oxygen species (ROS) also involved mitochondria-dependent signaling process is needed in hypoxia to promote HIF-1 $\alpha$ stabilization. Also, mitochondrial ROS is required for HIF- $1 \alpha$-DNA binding or induction of erythropoietin (EPO)-mediated HIF-1 $\alpha$ expression, VEGF and glycolytic enzyme. ROS increases angiogenesis by increasing HIF-1 $\alpha$ and activation and expression of VEGF reseptor-2 (VEGFR2) as illustrated in Figure 2. Oxidative stress also induces VEGF-independently angiogenesis by an oxidative phospholipid or post-translational modification and resulting in metabolite acting as a ligand. Survivin expression is a downstream target of important HIF-1 $\alpha$ because survivin function is needed to keep cell viability in the hypoxic condition. Thus, HIF- $1 \alpha$-dependent transcription of survivin can mediate the cell viability at low oxygen condition which due to tumor growth. Besides, increased survivin expression can contribute to VEGF synthesis in hypoxia by VEGF-A upregulation by HIF-1 $\alpha$ and HIF- $2 \alpha .^{4}$

\section{Survivin mechanism in STAT3}

STAT3 or 3' transcription activator is an oncogenic transcriptional factor involved in cytokine signaling and a group of E2 factor (E2F) transcriptional factor functions in G1/synthesis(S) transition of cell cycle. STAT3 activated by cytokine or growth factors in various types of cancer and play a significant role in tumor cell proliferation, differentiation and defense. STAT3 can translocate into the nucleus and regulate transcription of specific target gene like survivin..$^{30}$ In primary lymphoma cells, STAT3 signaling inhibition may trigger apoptosis via a mechanism involving survivin transcription suppression. Many further studies found that STAT3 directly binds survivin promoter and regulate the expression. Survivin protein sequences have a lysine-rich region at the C-terminal, forming a primary platform for proteins interaction and modification at post-transitional level. ${ }^{31}$

\section{Survivin surviving mechanism in Notch signaling pathway} Notch signaling is essential for cell development, differentiation, survival and proliferation of tissue morphogenesis. Impairment occurs in differentiation, survival regulation and or proliferation will cause transformation into malignant, so that pathophysiological Notch signal provides the contribution to cancer progression in some different ways. Notch signaling can regulate survivin expression, for example, Notch-1 signaling activated by 


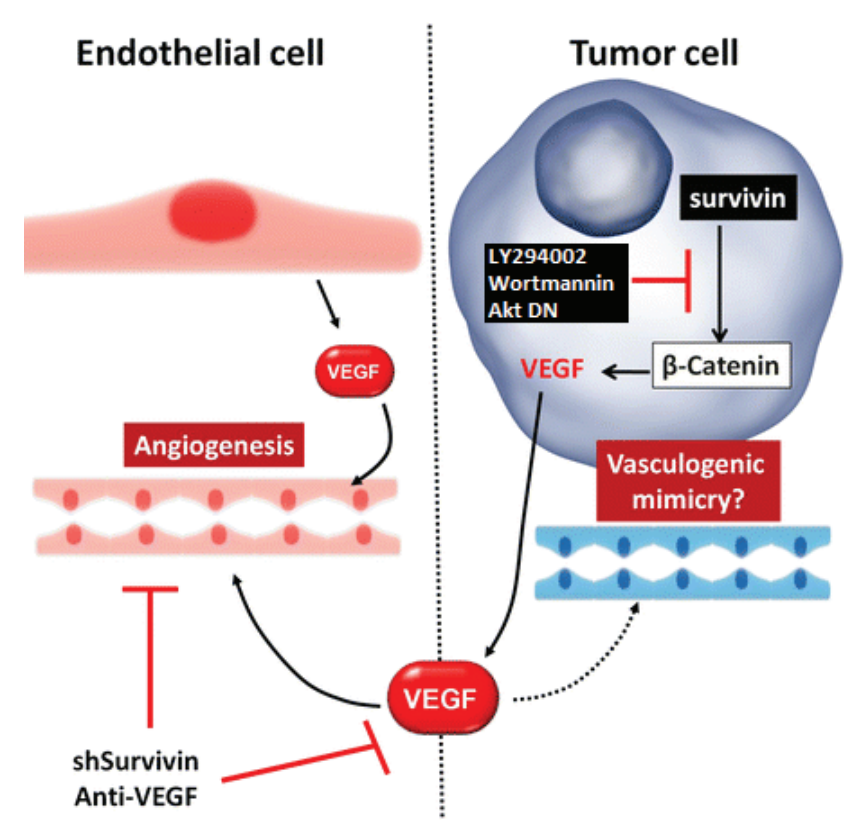

Figure 1. The survivin and VEGF connection through $\beta$-catenin pathway. ${ }^{4}$ (Adapted with permission from Springer Nature). shSurvivin: small hairpin Survivin, Akt-DN: Akt dominantnegative form.

survivin expression under the influence of hypoxia inside non-small cell lung cancer (NSCLC). Notch-1 signaling activation associated to increased HIF- $1 \alpha$, which functions as a co-activator via its interaction with the activated form of Notch-1, Notch 1 intracellular domain (N1ICD) that facilitate relationship between recombination signal binding protein for immunoglobulin kappa J region (RBPJ) in the survivin promoter. ${ }^{32}$

\section{Survivin mechanism in TGF- $\beta$ signaling}

Changes in cell behavior produce responsiveness not only from the genetic change like oncogenic activation or tumor suppressor gene inactivation, but also from altered production, or response to stimulation growth of differential factor. Among them, there is transforming growth factor beta (TGF- $\beta$ ) change and signal effector as the primary determinator of carcinoma cell behavior. Autocrine and paracrine effects of TGF- $\beta$ occur in the tumor cell and provide both positive and negative influences in the environment of cancer progression. Thus, TGF- $\beta$ signaling pathway plays roles as tumor pathway suppressor and progression promoter and tumor invasion.

TGF- $\beta$ and its signaling effectors such as similar to mothers against decapentaplegic $2(\operatorname{Smad} 2)$ and $\operatorname{Smad} 3$ proteins are key factors in proliferation-controlling and tumor cell differentiation. Smad2 and Smad3 are activated via carboxy-terminal phosphorylation by type 1 TGF- $\beta$

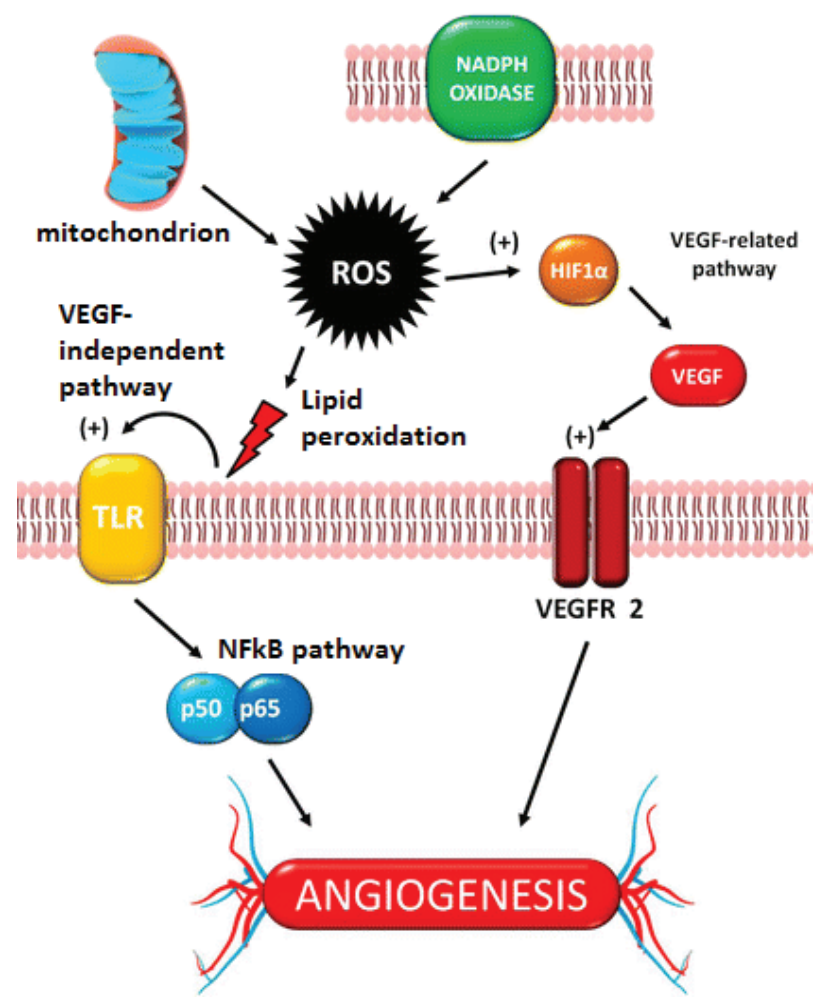

Figure 2. Upregulation of HIF-1 $\alpha$ induce angiogenesis through VEGF in hypoxia. ${ }^{4}$ (Adapted with permission from Springer Nature). NADPH: nicotinamide adenine dinucleotide phosphate, TLR: toll-like receptors..

kinase receptor and form a heterotrimeric complex with Smad4. The formed complex will translocate into the nucleus and acts as an activator of TGF- $\beta$-induced target gene transcription. TGF- $\beta$ is a tumor suppressor with its ability in cell growth inhibition via cell apoptosis.

TGF- $\beta$ identified as an important negative regulator of surviving. TGF- $\beta$ signaling suppression by either TGF- $\beta$ inhibitor receptor kinase or $\mathrm{Smad} 2$ and $\mathrm{Smad} 3$ silencing will induce survivin expression. ${ }^{33}$ On the contrary, survivin expression suppressed by TGF- $\beta$ signaling pathway at the transcriptional level via Smad2 and 3-dependent mechanism and cell cycle-dependent element/cell cycle genes homology region $(\mathrm{CDE} / \mathrm{CHR})$ from survivin promoter leading to retinoblastoma hypophosphorylation via Smad3-dependent mechanism. $^{34}$

\section{HPV infection and survivin expression relationship}

Is has been known that invasive cervical cancer is caused by HPV infection, especially HR-HPV type; mostly 16 and $18 .{ }^{35}$ Recent studies have shown that HPV-16 have an important role in cervical tumorigenesis related to survivin. ${ }^{36}$ The most 
important viral oncoprotein expressed by HPV-16 are E6 and E7 and have any effect on the expression of the human survivin gene in cervical cancer cell lines. HPV-16 E6 transactivates survivin promoter activity, and interestingly in the transient infection, it's transactivated all the survivin reporter constructs. However, in a cell line which no express $\mathrm{p} 53$, there is no transactivation effect on the survivin reporter constructs, this may suggest that $\mathrm{p} 53$ has a role in mediating transactivation effect of E6 oncoprotein to the survivin promoter. ${ }^{37}$ It well established that HR-HPV E6 oncoprotein induces the proteosome-mediated degradation of $\mathrm{p} 53,{ }^{38}$ as a logical implication that HPV E6 transactivates the survivin promoter mediated by the presence of $\mathrm{p} 53$ degradation. On the other hand, E6 oncoprotein also induces endogenous survivin transcription indicated by the high level of survivin mRNA. ${ }^{37}$

\section{Survivin as cervical cancer biomarker and target of therapy}

\section{Survivin as diagnostic marker}

Cervical cancer is well diagnosed today by Papanicolau staining and allowed to grading CIN and determining SCC along HPV DNA testing. Several findings concluded that survivin has an ability to do the same thing with Papanicolau staining for diagnosing cervical cancer. Survivin has gradual increase expression level in every stage of CIN and showing a gradual increase compared to the normal cervix, CIN and cervical SCC as well. ${ }^{9,39}$

$\mathrm{CIN}$ is a condition of precancerous squamous cell that has a risk of malignant progression, which is higher in the high level of CIN and well correlated with the expression of survivin in every stage of CIN. ${ }^{40,41}$ It undoubted that survivin would be a potential marker to predict clinical condition and risk for cancerous cervix tissue, showed by correlation of survivin expression with HR-HPV. It suggested that survivin has an ability to differentiate causal of cervical lesion whether HR-HPV or low-risk HPV (LR-HPV) but still cannot substitute HPV testing in monitoring risk of disease recurrence after treatment. ${ }^{35}$

The level of Survivin expression determined by immunostaining technique and polymerase chain reaction (PCR) that supposed to be more objective interpretation than conventional staining because it would be relatively close to the quantitative result. A new approach method in detection of survivin expression is a molecular beacon or tagging with fluorescent that suggested giving the objective results because of interpreted based on fluorescent intensity. ${ }^{42}$ As long as clinical research for survivin increased, more data collected can be more confidently propose survivin as a predictor for diagnosing cervical cancer and ability as a predictor, in combination with expression of p16 and phosphatase and tensin homolog (PTEN) would be more powerful. ${ }^{41}$ It is fascinating to make deeper exploration for survivin features in cervical cancer management. Another finding suggests that intracellular survivin well correlated to the progression of cervical cancer compared to nuclear survivin. ${ }^{43}$

\section{Survivin as prognostic marker}

Survivin expression recently was known have a close correlation with the occurrence, development and prognosis of cervical cancer. ${ }^{44}$ In most cervical cancer patients, survivin overexpression is correlated to poor survival rate and can predict aggression of cancerous tissue to the lymph node. The most correlated clinicopathological with the consistent result is clinical staging and lymph node metastasis, in contrast with Federation of Gynecology and Obstetrics (FIGO) stage, tumor size and age. Another data showed that high survivin is associated with reduced survival and supported by the clinical finding in the lung, gastric and esophageal cancer. ${ }^{45}$

Based on those results in the meta-analysis, survivin is suggested as a useful prognostic and tumor aggression marker for cervical cancer. Survivin also can predict the response of irradiation therapy as long as caspase- 3 an advanced stage cervical cancer. Survivin is known as caspase inhibitor that inhibits apoptosis so that over-expression of survivin increases tumor cell resistance to irradiation and hypoxia. ${ }^{5}$ Survivin in well correlated with hypoxia condition, most of the tumor cell makes their surrounding be hypoxia, and well-known marker for hypoxia is HIF- $1 \alpha$. Survivin expression regulated by HIF- $1 \alpha$ as a compensation mechanism of hypoxia; cell has to survive in hypoxic condition. The mechanism has enhanced the activity of HIF$1 \alpha$ on survivin promoter. ${ }^{29}$ So that no wonder if survivin can predict resistance to therapy and hypoxia in advanced cervical cancer. Surivivin is also applicable to the early stage of cervical cancer so that would be better management of patients. Survivin known to make cancer cell resistance to radiation therapy, as same as to hypoxic condition, it is a compensation mechanism or adaptation to stress. The cancerous cell has evaluated in response to radiation level; high intensity suggested to increase survivin expression and 
inhibit apoptosis so that radiation therapy efficacy would be not adequate. ${ }^{46}$

Based on the explanation above, there are other survivin features of effectiveness in the therapy of cervical cancer and to predict the intensity of radiation to keep tumor cells do not reach their adaptive response. Survivin also has an issue of cervical cancer resistance to chemotherapy. Survivin expression inversely proportional to sensitivity against cis-diamminedichloridoplatinum(II) (CDDP) and 5-fluorouracil (5-FU) but more clinical data required to encourage the power of survivin to predict resistance to chemotherapy. ${ }^{47}$

\section{Survivin as cervical cancer target therapy}

Resistance to therapy becomes a big problem in cancer management. Survivin known plays a significant role in resistance as described above. Survivin inhibits cell apoptosis and as a consequence, the tumor grows rapidly and increasing expression of survivin is correlated to therapy resistance. Simply, survivin would be a target and biomarker for efficacy due to its features in clinicopathological and progression of cervical cancer. ${ }^{48}$ In recent years, considerable efforts have been made to validate survivin as a preferred target and prognostic marker in cancer therapy. Some studies have addressed the effects of interfering survivin expression, or in tumor cell function; for instance, introduction a dominant negative variant of survivin into cervical cancer cells caused the multipolar mitotic spindles, cytokinesis failure, the formation of multinucleated cells, reduced proliferation and induction of apoptosis. ${ }^{49}$

In short, all of the approaches to survivin as a therapeutic target for cancer is interfering the biogenesis and function of survivin. Those strategies are started from inhibition of promoter or transcription factors, interfering transcripted mRNA that reduced translation grouped into nucleic acid base therapy, altering protein stability and posttranslational modification including protein folding and even more secondary modification and the most promising and save techniques are immunotherapy and genome editing. $., 7,47$

\section{Inhibition of survivin transcription}

Some transcription factors are known to recognize the specific response elements of survivin gene promoter and involved in the regulation of survivin mRNA regulation transcription. Thus, blocking the transcription of the survivin through inhibition of specific transcription factors suggested being a promising concept to interfere with survivin expression and function. ${ }^{49}$ Inhibitor transcription factor that selected from the compound library as an inhibitor of survivin promoter-reporter gene construct is sepantronium bromide (YM155). ${ }^{50,51}$ YM155 is the first small chemical molecule and known as a high selectivity inhibitory ability to survivin transcription with no activity against other anti-apoptotic molecules like cellular IAP (cIAP), XIAP or cIAP1. ${ }^{52}$ YM155 decreased survivin expression by repression of survivin promoter activity by binding to the transcription factor interleukin enhancerbinding factor 3 (ILF3) and disrupting ILF/p54 complex. YM155 effectively reduced expression of survivin and also reduced lymph node metastasis in cervical cancer. ${ }^{13}$

Another strategy in inhibiting survivin expression is interfering transcripted mRNA utilization including antisense oligonucleotide (AO), small interfering RNA (siRNA) and miRNA. AO are short single stranded RNA or DNA that complementary to as specific target mRNA which is survivin mRNA caused unable to translate into protein or even inhibiting at the protein level. The first attempt at AO therapy targeting survivin induced apoptosis in human cell melanoma cell lines and continued with another type of cancer such as lung, lymphoma, thyroid, head and neck cancer and cervical cancer as well..$^{51,53}$

The other promising approach in nucleotide-based survivin targeting is RNA interference (RNAi) such as siRNA which inhibits tumor growth by triggering apoptosis and commonly use for in vitro investigation to transfect to the survivin RNA. However, it has several limitations including disadvantages pharmacokinetic profile and ineffectively transfection into the cell and not affect a broad range of regulation compared to miRNA, so that miRNA more promising due to its ability to repress gene expression. An artificial miRNA has been developed to treat cervical cancer and show a good performance to arresting the cell cycle in $\mathrm{G} 2 / \mathrm{M}$, suppress survivin expression and increasing p53/p21 protein level..$^{55}$ Among nucleotide therapeutic strategies, miRNA show a reliable result to repress survivin expression and its function to treat cervical cancer, but all of the approaches need to be established transfection method and it seems not practical.

\section{Inhibition survivin at the post-translational level}

The distinguishable function of survivin in cell activity most likely realized through the domain structure of the protein molecule and regulated by subsequent modifications such 
as targeting enzyme that may interfere the process, altering folding and destabilizing survivin structure, or inhibiting phosphorylation of survivin. ${ }^{49}$ Based on the molecular pathway in nature of survivin, phosphorylation is the most common modification and play a critical role in survivin activity. For example, survivin phosphorylated at Thr117 by Aurora-B kinase and at Thr34 by polo-like kinase 1 (PLK1), both of that phosphorylation required for the correct spindle microtubule attachment. Thus, by inhibiting those enzymes, survivin activity may decrease and apoptosis will occur in the cancer cell. ${ }^{49,55}$

The other strategy is inhibiting survivin at the posttranslational level through the inhibiting agent to CDK and heat shock protein 90 (Hsp90). Inhibiting CDK would repress survivin phosphorylation. Thus survivin will not be active and inhibiting Hsp90 would prevent survivin to make a complex with Hsp90. Shepherdin is a cell-permeable antagonist of the Hsp90 and inhibits its function by competing with adenosine triphosphate (ATP) binding. ${ }^{51}$ Destabilizing protein structure of survivin has known through Kirsten rat sarcoma viral oncogene homolog (K-Ras) mutation. Mutant K-Ras decrease survivin levels through ubiquitination and proteasomal degradation. ${ }^{56}$ There is no sufficient data in treating cervical cancer using a post-translational modification of survivin but based on explained pathway above, we can see that mechanism of every cancer is same all the way, so that this approach also have a contribution in treating cervical cancer.

\section{Immunotherapy and vaccine}

The immune system of human broadly divided into two groups, which is innate and adaptive immunity and further, adaptive immunity consists of cell-mediated immunity and humoral immunity. Adaptive immunity has been explored to become a potential approach in immune therapy for malignancy, especially cervical cancer. The fundamental approach based on specific recognition of tumor-associated antigen by cytotoxic T lymphocytes (CTL) which activated by presented dendritic cells. ${ }^{57,58}$ About $30 \%$ of cervical cancer patients will experience failure therapy including surgery, radiotherapy and chemotherapy, as described above, survivin have an important role in that condition..$^{59}$ For that, targeting survivin immunological is very promising for treating cervical cancer and another cancer because of survivin suggested being a universal antigen for tumor based on the first evidence that survivin can induce specific CTL in vitro when the protein processed and presented by dendritic cells. ${ }^{60}$ Cancer cells containing a lot of survivin including cervical cancer cells, after survivin epitopes recognized by CTL and then neutralized, the number of survivin decrease and apoptosis of cancer cell not inhibited. While CTL response is powerful as anti-tumor targeting agents, CTL response has not led to consistent clinical responses, so that some studies have evaluated the value of $\mathrm{CD} 4^{+} \mathrm{T}$-cells, once $\mathrm{CD}^{+}$cells have activated. The release of many cytokines and enhanced CTL immune response, therefore, a vaccine strategy that combining CTL and $\mathrm{CD}^{+}$cells improved survivin targeted vaccination approach. ${ }^{10}$ Immunotherapy for cervical cancer suggested to non-invasive way with good efficacy compared to chemotherapy or radiotherapy that known non-selective and made patients more suffer due to their side effects.

\section{Gene therapy}

The last strategy and still become a field of interest and the most promising, which is gene therapy. There are two main approaches have been developed for targeting survivin with gene editing. One based on the activation of apoptosis in tumor cell infected with survivin-containing vector and expression of cytotoxic gene driven by survivin promoter in the tumor cell. ${ }^{61}$ An example of essential gene therapy has transfected a plasmid encoding a nonphosphorylated Thr34 mutated to alanine using replication-deficient adenovirus; this approach is selective and efficient to repress survivin in HeLa cell line and application at dysregulated apoptotic balance in cervical cancer patients. ${ }^{62}$ Recent development in gene therapy is genome editing. This approach is more promising and safer than the previous methods. There are three genome-editing techniques have been developed today; zinc-finger nucleases (ZFNs), transcription activatorlike effector nucleases (TALENs) and clustered regularly interspaced short palindromic repeats-associated protein- 9 nuclease (CRISPR/Cas-9). ${ }^{51}$ To date, CRISPR/Cas9 system suggested to be a versatile system for genome editing, in the case of cancer, survivin has a high expression that inhibits apoptosis, suppressing the expression of survivin would be more easy, high specificity and less side effect.

Recent findings show that CRISPR/Cas9 application to fight cervical cancer started from the beginning; HPV infection to the therapy after cancer promoted. In one study, HeLa and SiHa cervical cancer contained HPV18 and HPV16 genome. Inactivating deletion and insertion 
mutations were induced in E6 and E7 resulting activation of p53 and resulting cell cycle arrest..$^{63}$ Due to arresting cell cycle ability, survivin has also involved in that process so that survivin expression would repress as well after Cas9 introduction. There is no sufficient data in application genome editing to cervical cancer patients to date, but based on its performance and all of the advantages, cervical cancer treatment has a light of hope at the end of a tunnel.

\section{Conclusion}

Cervical cancer is one of cancer promoted by virus infection especially HR-HPV type 16 and 18 expressed E6 oncoprotein. The unique link between HPV infection with survivin expression and regulation is fascinating. Survivin plays a significant role in every step of cervical and other cancer progressions through standard mechanisms. Survivin has many features in cervical cancer management, started from diagnosis, prognosis and therapy.

\section{References}

1. NCI Dictionary of Cancer Terms [Internet]. National Cancer Institute. 2016 [cited 12 December 2016]. Available from: https://www. cancer.gov/publications/dictionaries/cancer-terms?cdrid=560661

2. Schiffman M, Castle P, Jeronimo J, Rodriguez A, Wacholder S. Human Papillomavirus and Cervical Cancer. The Lancet. 2007; 370(9590): 890-907.

3. Sahasrabuddhe V, Luhn P, Wentzensen N. Human Papillomavirus and Cervical Cancer: Biomarkers for Improved Prevention Efforts. Future Microbiology. 2011; 6(9): 1083-98.

4. Sanhueza C, Wehinger S, Castillo Bennett J, Valenzuela M, Owen GI, Quest AFG. The Twisted Survivin Connection to Angiogenesis. Mol Cancer. 14: 198. doi: 10.1186/s12943-015-0467-1

5. Kusuma F, Nuranna L, Rustamadji PD, Sutrisna B. Caspase 3 and Survivin Expressions as a Predictor of Response to Radiation Therapy in Advanced Stage Cervical Cancer. Indones J Obstet Gynecol. 2012; 35(3): 139-45.

6. Garg H, Suri P, Gupta JC, Talwar GP, Dubey S. Survivin: a Unique Target for Tumor Therapy. Cancer Cell Int. 2016; 16: 49. doi: 10.1186/s12935-016-0326-1

7. Chen X, Duan N, Zhang C, Zhang W. Survivin and Tumorigenesis: Molecular Mechanisms and Therapeutic Strategies. J Cancer. 2016; 7(3): 314-23.

8. Fernández JG, Rodríguez DA, Valenzuela M, Calderon C, Urzúa U, Munroe D, et al. Survivin Expression Promotes VEGF-induced Tumor Angiogenesis via PI3K/Akt Enhanced $\beta$-Catenin/TcfLef Dependent Transcription. Mol Cancer. 2014; 13: 209. doi: 10.1186/1476-4598-13-209

9. Liu HQ, Wang YH, Wang LL, Hao M. P16INK4A and Survivin: Diagnostic and Prognostic Markers in Cervical Intraepithelial Neoplasia and Cervical Squamous Cell Carcinoma. Exp Mol Pathol. 2015; 99(1): 44-9.

10. Ciesielski MJ, Qiu J, Fenstermaker RA. Survivin as a Cancer
Vaccine Target. J Vaccines Vaccin. 2014; 5: 3. doi: 10.4172/21577560.1000230

11. Altieri DC. Survivin, Cancer Networks and Pathway-Directed Drug Discovery. Nat Rev Cancer. 2008; 8(1): 61-70.

12. Kogo R, How C, Chaudary N, Bruce J, Shi W, Hill RP, et al. The microRNA-218 Survivin Axis Regulates Migration, Invasion, and Lymph Node Metastasis in Cervical Cancer. Oncotarget. 2015; 6(2): 1090-100.

13. Sandra F, Khosravi-Far R. Phosphorylated-Survivin at Ser81 Induced Protein Kinase A (PKA): A Back Loop. Indones Biomed J. 2011; 3(2): 138-42.

14. Sandra F, Khosravi-Far R. Ser81 Survivin Induced Protein Kinase A (PKA)-dependent Phosphatidylinositol 3-kinase (PI3K) Activity. Indones Biomed J. 2014; 6(3): 157-62.

15. Barrett RM, Osborne TP, Wheatley SP. Phosphorylation of Survivin at Threonine 34 Inhibits Its Mitotic Function and Enhances Its Cytoprotective Activity. Cell Cycle. 2009; 8(2): 278-83.

16. Sandra F, Khosravi-Far R. Survivin S81A Enhanced TRAIL's Activity in Inducing Apoptosis. Indones Biomed J. 2010; 2(3); 113-7.

17. Sukpan K, Settakorn J, Khunamornpong S, Cheewakriangkrai C, Srisomboon J, Siriaunkgul S. Expression of Survivin, CD117, and C-erbB-2 in Neuroendocrine Carcinoma of the Uterine Cervix. Int J Gynecol Cancer. 2011; 21(5): 911-7.

18. Li Q, Zhao J, Liu J, Jia L, Huang H, Xu Y, et al. Survivin Stable Knockdown by siRNA Inhibits Tumor Cell Growth and Angiogenesis in Breast and Cervical Cancers. Cancer Biol Ther. 2006; 5(7): 860-66.

19. Li F, Ackermann EJ, Bennett CF, Rothermel AL, Plescia J, Tognin $\mathrm{S}$, et al. Pleiotropic Cell-division Defects and Apoptosis Induced by Interference with Survivin Function. Nat Cell Biol. 1999; 1(8): 461-6.

20. Lippert BM, Knauer SK, Fetz V, Mann W, Stauber RH. Dynamic Survivin in Head and Neck Cancer: Molecular Mechanism and Therapeutic Potential. Int J Cancer. 2007; 121(6): 1169-74.

21. Mita AC, Mita MM, Nawrocki ST, Giles FJ. Survivin: Key Regulator of Mitosis and Apoptosis and Novel Target for Cancer Therapeutics. Clin Cancer Res. 2008; 14(16): 5000-5.

22. Unruhe B, Schroder E, Wunsch D, Knauer S. An Old Flame Never Dies: Survivin in Cancer and Cellular Senescence. Gerontology. 2015; 62(2): 173-81.

23. Beardmore VA, Ahonen LJ, Gorbsky GJ, Kallio MJ. Survivin Dynamics Increases at Centromeres during G2/M Phase Transition and is Regulated by Microtubule-attachment and Aurora B Kinase Activity. J Cell Sci. 2004; 117: 4033-42.

24. Papanikolaou V, Iliopulos D, Dimou I, Dubos S, Kappas C, KitsiouTzeli S, et al. Survivin Regulation by HER2 Through NF-kappaB and c-myc in Irradiated Breast Cancer Cells. J Cell Mol Med. 2011; 15(7): 1542-50.

25. Mehrotra S, Languino LR, Raskett CM, Mercurio AM, Dohi T, Altieri DC. IAP regulation of metastasis. Cancer Cell. 2010, 17(1): 53-64.

26. Komiya Y, Habas R. Wnt Signal Transduction Pathways. Organogenesis. 2008; 4(2): 68-75.

27. Tapia JC, Torres VA, Rodriguez DA, Leyton L, Quest AF. Casein Kinase 2 (CK2) Increases Survivin Expression via Enhanced Beta-catenin-T cell Factor/Lymphoid Enhancer Binding Factordependent Transcription. Proc Natl Acad Sci USA. 2006; 103(41): 15079-84.

28. Penn JS, Madan A, Caldwell RB, Bartoli M, Caldwell RW, Hartnett ME. Vascular Endothelial Growth Factor in Eye Disease. Prog 
Retin Eye Res. 2008; 27(4): 331-71.

29. Bai H, Ge S, Lu J, Qian G, Xu R. Hypoxia Inducible Factor-1 $\alpha-$ mediated Activation of Survivin in Cervical Cancer Cells. J Obstet Gynaecol Res. 2012; 39(2): 555-63.

30. Carpenter RL, Lo HW. STAT3 Target Genes Relevant to Human Cancers. Cancers (Basel). 2014; 6(2): 897-925.

31. Wang H, Holloway MP, Ma L, Cooper ZA, Riolo M, Samkari A, et al. Acetylation Directs Survivin Nuclear Localization to Repress STAT3 Oncogenic Activity. J Biol Chem. 2010; 285(46): 36129-37.

32. Chen Y, Li D, Liu H, Xu H, Zheng H, Qian F, et al. Notch-1 Signaling Facilitates Survivin Expression in Human Non-small Cell Lung Cancer Cells. Cancer Biol Ther. 2011; 11(1): 14-21.

33. Derynck R, Akhurst RJ, Balmain A. TGF-beta Signaling in Tumor Suppression and Cancer Progression. Nat Genet. 2001; 29(2): 11729.

34. Song K, Shankar E, Yang J, Bane KL, Wahdan-Alaswad R, Danielpour D. Critical Role of a Survivin/TGF- $\beta /$ mTORC1 Axis in IGF-I-mediated Growth of Prostate Epithelial cells. PLoS One. 2013; 8(5): e61896. doi: 10.1371/journal.pone.0061896

35. Branca M, Giorgi C, Santini D, Di Bonito L, Ciotti M, Costa S, et al. Survivin as a Marker of Cervical Intraepithelial Neoplasia and HighRisk Human Papillomavirus and a Predictor of Virus Clearance and Prognosis in Cervical Cancer. Am J Clin Pathol. 2005; 124(1): 11321.

36. Li F. Role of Survivin and Its Splice Variants in Tumorigenesis. Br J Cancer. 2005; 92(2): 212-6.

37. Borbely AA, Murvai M, Kónya J, Beck Z, Gergely L, Li F, et al. Effects of Human Papillomavirus Type 16 Oncoproteins on Survivin Gene Expression. J Gen Virol. 2006; 87(2): 287-94.

38. Münger K, Howley PM. Human Papillomavirus Immortalization and Transformation Functions. Virus Res. 2002; 89(2): 213-28.

39. Grigore M, Teleman S, Ungureanu D, Mares A. Molecular Markers in Cervical Screening - a Promise for the Future. Romanian Rev Lab Med. 2013; 21(2): 231-9.

40. Wu SF, Zhang JW, Qian WY, Yang YB, Liu Y, Dong Y, et al. Altered Expression of Survivin, Fas and FasL Contributed to Cervical Cancer Development and Metastasis. Eur Rev Med Pharmacol Sci. 2012; 16(15): 2044-50.

41. Lu D, Qian J, Yin X, Xiao Q, Wang C, Zeng Y. Expression of PTEN and Survivin in Cervical Cancer: Promising Biological Markers for Early Diagnosis and Prognostic Evaluation. Br J Biomed Sci. 2012; 69(4):143-6.

42. Xue Y, An R, Zhang D, Zhao J, Wang X, Yang L, et al. Detection of Survivin Expression in Cervical Cancer Cells using Molecular Beacon Imaging: New Strategy for the Diagnosis of Cervical Cancer. Eur J Obstet Gynecol Reprod Biol. 2011; 159(1): 204-8.

43. Kim S, Hong R. Significance of Intracellular Localization of Survivin in Cervical Squamous Cell Lesions: Correlation with Disease Progression. Oncol Lett. 2014; 7(5): 1589-93.

44. Cao XQ, Lu HS, Zhang L, Chen LL, Gan MF. MEKK3 and Survivin Expression in Cervical Cancer: Association with Clinicopathological Factors and Prognosis. Asian Pac J Cancer Prev. 2014; 15(13): 5271-6.

45. Cheng KY, Wang ZL, Gu QY, Hao M. Survivin Overexpression is Associated with Aggressive Clinicopathological Features in Cervical Carcinoma: A Meta-Analysis. PLOS ONE. 2016; 11(10): e0165117. doi: 10.1371/journal.pone.0165117

46. Grdina DJ, Murley JS, Miller RC, Mauceri HJ, Sutton HG, Li JJ, et al. A Survivin-associated Adaptive Response in Radiation Therapy. Cancer Res. 2013; 73(14): 4418-28.

47. Saxena A, Yashar C, Taylor DD, Gercel-Taylor C. Cellular Response to Chemotherapy and Radiation in Cervical Cancer. Am J Obstet Gynecol. 2005; 192(5): 1399-403.

48. Iida M, Banno K, Yanokura M, Nakamura K, Adachi M, Nogami Y, et al. Candidate Biomarkers for Cervical Cancer Treatment: Potential for Clinical Practice (Review). Mol Clin Oncol. 2014; 2(5): 647-55.

49. Groner B, Weiss A. Targeting Survivin in Cancer: Novel Drug Development Approaches. BioDrugs. 2014; 28(1): 27-39.

50. Nakahara T, Kita A, Yamanaka K, Mori M, Amino N, Takeuchi M, et al. YM155, a Novel Small-molecule Survivin Suppressant, Induces Regression of Established Human Hormone-refractory Prostate Tumor Xenografts. Cancer Res. 2007; 67(17): 8014-21.

51. Mobahat M, Narendran A, Riabowol K. Survivin as a Preferential Target for Cancer Therapy. Int J Mol Sci. 2014; 15(2): 2494-516.

52. Wang L, Waltenberger B, Pferschy-Wenzig EM, Blunder M, Liu $\mathrm{X}$, Malainer $\mathrm{C}$, et al. Natural Product Agonists of Peroxisome Proliferator-activated Receptor Gamma (PPAR $\gamma$ ): a Review. Biochem Pharmacol. 2014; 92(1): 73-89.

53. Carrasco RA, Stamm NB, Marcusson E, Sandusky G, Iversen P, Patel BKR. Antisense Inhibition of Survivin Expression as a Cancer Therapeutic. Mol Cancer Ther. 2011; 10(2): 221-32.

54. Chi Y, Wang X, Yang Y, Zhang C, Ertl HCJ, Zhou D. Survivintargeting Artificial MicroRNAs Mediated by Adenovirus Suppress Tumor Activity in Cancer Cells and Xenograft Models. Mol Ther Acids. 2014; 3(11): e208. doi: 10.1038/mtna.2014.59

55. Chu Y, Yao PY, Wang W, Wang D, Wang Z, Zhang L, et al. Aurora B Kinase Activation Requires Survivin Priming Phosphorylation by PLK1. J Mol Cell Biol. 2011; 3(4): 260-7.

56. Tecleab A, Sebti SM. Depletion of K-Ras Promotes Proteasome Degradation of Survivin. Cell Cycle Georget Tex. 2013; 12(3): 52232.

57. Schmidt SM, Schag K, Müller MR, Weck MM, Appel S, Kanz L, et al. Survivin is a Shared Tumor-Associated Antigen Expressed in a Broad Variety of Malignancies and Recognized by Specific Cytotoxic T Cells. Blood. 2003; 102(2): 571-6.

58. Menderes G, Black J, Schwab CL, Santin AD. Immunotherapy and Targeted Therapy for Cervical Cancer: an Update. Expert Rev Anticancer Ther. 2016; 16(1): 83-98.

59. Lin CT, Wang CN, Lai CH. Immunotherapy for Advanced or Relapsed Cervical Cancer. Gynecol Minim Invasive Ther. 2013; 2(1): 3-7.

60. Andersen MH, Straten PT. Survivin-A Universal Tumor Antigen. Histol Histopathol. 2002; 17(2): 669-75.

61. Pennati M, Folini M, Zaffaroni N. Targeting Survivin in Cancer Therapy: Fulfilled Promises and Open Questions. Carcinogenesis. 2007; 28(6): 1133-9.

62. Mesri M, Wall NR, Li J, Kim RW, Altieri DC. Cancer Gene Therapy Using a Survivin Mutant Adenovirus. J Clin Invest. 2001; 108(7): 981-90.

63. White MK, Hu W, Khalili K. The CRISPR/Cas9 Genome Editing Methodology as a Weapon Against Human Viruses. Discov Med. 2015; 19(105): 255-62. 